\title{
The Complexity of the Identification of Burkholderia cepacia Strain Which Caused Septicemia
}

\author{
Lan Anh Thi Bui ${ }^{1}$, Irina Zakharova ${ }^{2,}{ }^{*}$, Ivan Shpak ${ }^{2}$, Natalya Teteryatnikova ${ }^{2}$, Dimitriy Ustinov ${ }^{2}$, Yulia \\ Kuzyutina ${ }^{2}$, Ha Ngoc Nguyen ${ }^{3}$ and Dmitry Viktorov ${ }^{2}$ \\ ${ }^{1}$ Department of Tropical Medicine, Russian-Vietnamese Tropical Research Center, Hanoi, Vietnam \\ ${ }^{2}$ Department of Microbiology, Volgograd Plague Control Research Institute, Volgograd, Russia \\ ${ }^{3}$ Phu Tho Provincial General Hospital, Viet Tri, Vietnam \\ "Corresponding author: Department of Microbiology, Volgograd Plague Control Research Institute, Volgograd, Russia. Email: zib279@gmail.com
}

Received 2018 August 01; Revised 2018 October 18; Accepted 2018 October 20.

\begin{abstract}
Background: The correct identifying of pathogenic Burkholderia spp. using available commercial biochemical systems is a certain problem due to metabolic plasticity and variable enzymatic profile of isolates.

Objectives: The current study aimed at using specific PCR and conventional multi-locus sequence typing (MLST) scheme to confirm the uncertain identification results for Burkholderia cepacia clinical isolate.

Methods: Multilocus species-specific PCR and MLST profiling of high-throughput sequencing data have been used to clarify the varied results of biochemical identification of the strain.

Results: The strain isolated from a patient with septicemia was initially identified as B. pseudomallei by Vitek 2 GN system but has an uncharacteristic antimicrobial resistance pattern and colony morphology. A species-specific multilocus PCR and whole-genome sequence profiling, according to the MLST scheme, allowed to identify an isolate as B. cepacia.

Conclusions: The obtained results demonstrate the preference of molecular tests for correct identifying of pathogenic Burkholderia, considering that misidentification of those may lead to improper treatment or increase of biosafety risk.
\end{abstract}

Keywords: Burkholderia cepacia, B. pseudomallei, Sepsis, PCR, Whole Genome Sequencing, Molecular Typing

\section{Background}

The genus Burkholderia is characterized by taxonomic complexity and includes such highly pathogenic species as Burkholderia pseudomallei and B. mallei, the causative agents of melioidosis and glanders, respectively, and opportunistic pathogens of B. cepacia complex. Burkholderia cepacia complex as well as $B$. pseudomallei are highly resistant to antimicrobials, however, they have different resistance patterns (1). Therefore, the correct and welltimed identification of these pathogens is urgent for successful treatment. Furthermore, the wrong identification of highly pathogenic bacteria increases the risks for laboratory stuff due to potential unsafe manipulation. The currently available biochemical systems widely used in clinical microbiology laboratories can misidentify some Burkholderia species. The certain problem is associated with the misidentification of B. pseudomallei as B. cepacia and vice versa using Vitek 2 platform (bioMerieux, France) $(2,3)$.

\section{Objectives}

This work aimed to clarify the results of identification of clinical Burkholderia spp. isolate using species-specific PCR and MLST profiling of whole-genome sequence data.

\section{Methods}

\subsection{Case Description}

A case of septicemia with severe fever in a 68-year-old woman was preliminarily diagnosed as 'melioidosis' in Phu Tho Provincial General Hospital in January 2018. In anamnesis, there were no risk factors such as diabetes, AIDS, cystic fibrosis, or immune system diseases.

\subsection{Isolation and Identification of the Strain}

A non-fermenting Gram-negative rod was isolated from the blood of a patient. The strain was subcultured on LB agar (HiMedia Laboratories Pvt. Ltd, India) for 24 hours at $37^{\circ} \mathrm{C}$ before testing with the Vitek 2 GN cards 
(bioMerieux, France), according to the manufacturer's instructions. The antibiotic susceptibility was tested according to CLSI recommendation (4).

\subsection{Molecular Methods}

The multi-locus PCR assay targeting an individual set of chromosomal beta-lactamase genes in B.pseudomallei and $B$. cepacia complex was performed as described previously (5). Genomic DNA of the strain was isolated using GeneJET genomic DNA purification kit (Thermo Scientifics, Lithuania). The pair-end library was constructed via Nextera XT library preparation kit (Illumina Inc., USA). Sequencing was performed on the Illumina MiSeq platform (Illumina Inc., USA).

\subsection{Bioinformatics Tool}

Filtered reads were assembled de novo using SPAdes 3.11 software (6). The sequences of seven house-keeping genes, according to the MLST scheme by Spilker et al. (7), were matched in assembly using the BLASTn algorithm. Multiple alignments and phylogenetic analysis were performed using the MEGA 7 package (8). Identification of related genotypes in the MLST dataset was performed using eBURST v3 algorithm (http://eburst.mlst.net/).

\section{Results}

The isolated strain was identified initially as B. pseudomallei using Vitek 2 GN (bioMerieux, France) with a reliability of $87 \%$. The strain was resistant to gentamicin, colistin, and amoxicillin/clavulanate. It was also intermediate resistant to imipenem and was sensitive to ceftazidime, meropenem, doxycycline, and co-trimoxazole. The observed amoxicillin/clavulanate resistance pattern and colony morphology on Ashdown agar cast doubt on the results of biochemical identification. The strain was additionally tested using a recently developed multiplex PCR for detection of an individual set of chromosomal class $B$ and D beta-lactamase genes in B. pseudomallei and B. cepacia complex (5). The analysis suggested that the isolate belongs to B. cepacia. The strain was designated as B. cepacia PT02. Three months later of initial biochemical testing, the strain was identified as B. cepacia by Vitek 2 with a reliability of $91 \%$.

Whole-genome sequencing of B. cepacia PT02 was carried out for final confirmation of obtained results. The obtained contigs were deposited in the Genbank database (accession no. QLUZ00000000). The established MLST profile of the isolate was added to PubMLST B. cepacia complex database (https://pubmlst.org/bcc) with ID 2680. The MLST analysis revealed new variants of gltB and gyrB alleles, which were not previously described. The resulting allelic profile of B. cepacia PT02 was assigned to ST1400, which was not presented at the time in Pub MLST. The rMLST profiling of PT02 revealed the concordance in 42 out of 53 loci used in the database.

According to Maximum Likelihood phylogeny (bootstrap $=1000$ ), the PT02 isolate belongs to the $B$. cepacia clade of the tree (Figure 1). The similar results were obtained by eBURST analysis of allelic profiles of PT02 and 2688 B. cepacia complex strains from the MLST database. The PT02 isolate also belonged to B. cepacia branch and joined the clonal complex of B. cepacia CVS136 (Figure 2). The root strain CVS136 (Taiwan) and strain R-66653 (Belgium) were most closely related to PT02 (the genetic distance of 0.43 and 0.57 , respectively). It should be noticed that all strains of the CVS136 clonal complex were isolated from patients or hospital environment. Thus, B. cepacia R52513 and R-66653 were isolated from non-cystic fibrosis patient (NON) in Belgium, B. cepacia CEP0615-from cystic fibrosis patient in the USA (CF), and B. cepacia NML171677from non-cystic fibrosis patient with septicemia in Canada (NON, blood culture).

\section{Discussion}

The significant phenotypic variability is common in B. cepacia and other B. cepacia complex species. In addition, there has been controversy regarding the optimal differentiation system for B. pseudomallei complex and B. cepacia complex species. Although conventional tests (colony morphology on selective media, antimicrobial resistance patterns, agglutination) usually give acceptable discrimination, they are time-consuming. The misidentification of $B$. pseudomallei as B. cepacia and vice versa by commonly used Vitek 2 system was repeatedly described (3, 9, 10). Recently, Podin et al. noted that two enzymatic tests, NAGA (beta-N-acetyl-galactosaminidase) and BNAG (beta-N-acetyl-glucosaminidase), were distinct between correctly and misidentified B. pseudomallei isolates (11). In addition, the combinations of positive dCEL (Dcellobiose) and negative ProA (L-proline arylamidase), TyrA (tyrosine arylamidase), or NAGA were observed in B. pseudomallei strains incorrectly identified as B. cepacia (12).

The $B$. cepacia PT02 described here was initially positive for dCEL and TyrA, which leads to misidentification of the isolate as B. pseudomallei on Vitek 2 platform. Subsequently, the negative TyrA test, after a three-month storage period, was a key biochemical feature to identify the isolate as B. cepacia. The metabolic plasticity and variable enzymatic profile complicate the reliable biochemical differentiation within B. cepacia complex species, as well as from other Burkholderia spp. (13). Currently, this is the main reason why the molecular methods have a preferential use for B. cepacia complex differentiation. To date, there is a wide 


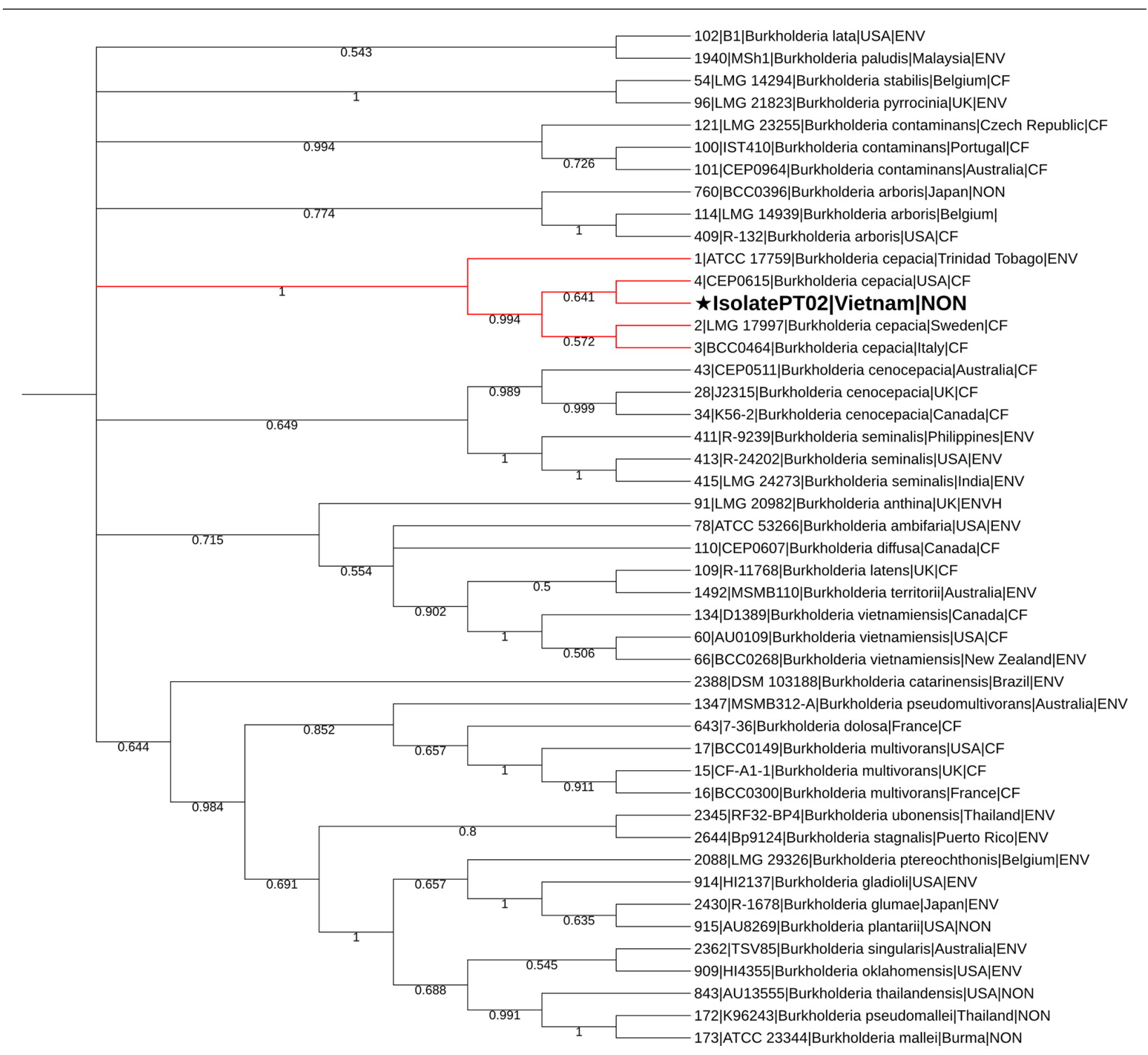

Figure 1. The genetic distance of 45 concatenated sequences of Burkholderia spp., and B. cepacia PT02 house-keeping genes according to MLST scheme (7). Maximum Likelihood dendrogram (bootstrap $=1000$ ) constructed using the TamuraNei model. The consensus bootstrap tree was obtained by 1000 iterations to demonstrate the reliability of clade forming. The multiple alignments were performed using MUSCLE algorithm. Alignment blocks containing gaps and missing data were excluded from analysis. The final dataset contained 2,271 sites.

range of PCR-based methods developed for rapid identification of $B$. cepacia complex species $(14,15)$ and their differentiation from $B$. pseudomallei complex $(5,16,17)$. The described detection of a $B$. cepacia complex specific set of beta-lactamase genes and sequence type determination allowed us to confirm the studied isolate decisively as B. cepacia.

\subsection{Conclusions}

The complex application of various molecular methods, considering their growing availability and high dis- criminatory power, in some cases can provide the most accurate and rapid identification of pathogenic Burkholderia, and thus, reduce risks of improper treatment or biosafety threat. The data mentioned above also demonstrates a better resolution of high-throughput sequencing/in silico MLST vs. conventional B. cepacia complex genomovar determination by recA restriction fragment length polymorphism. 


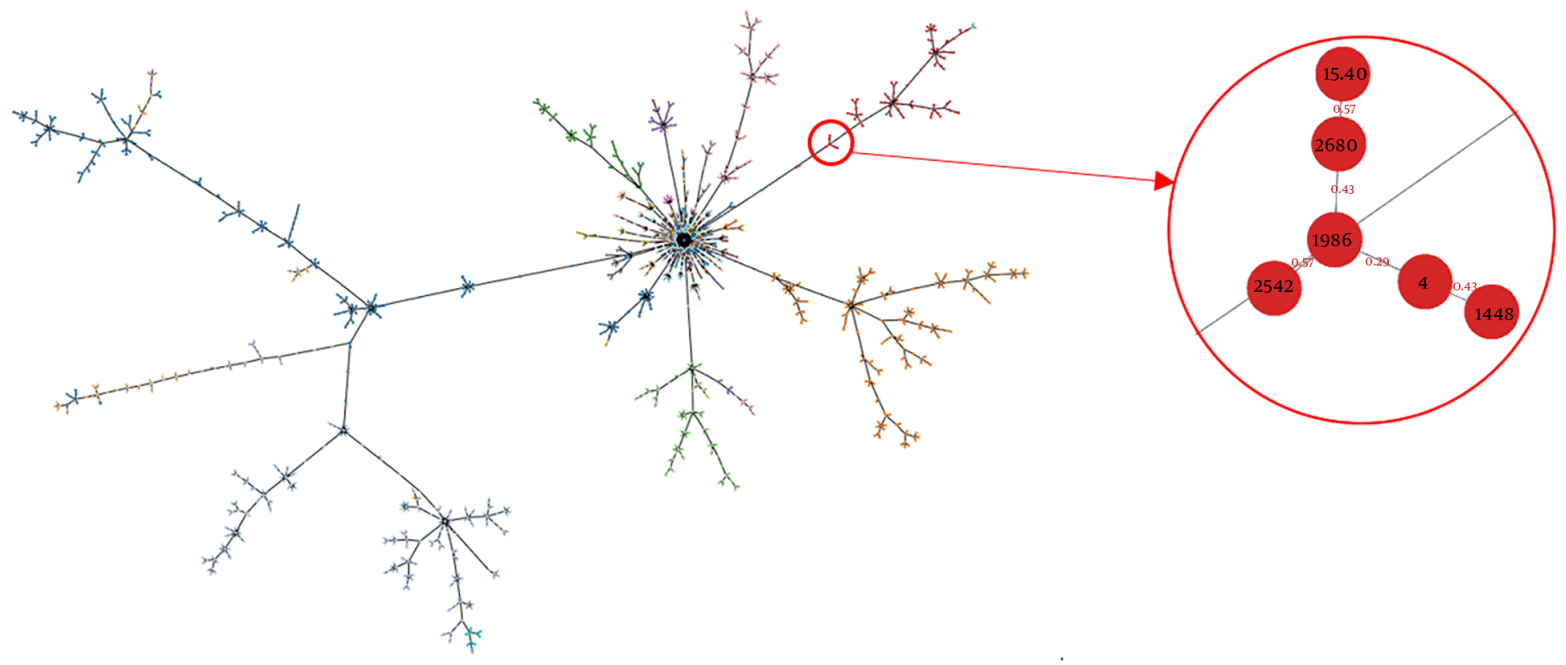

Figure 2. Population snapshot of 2688 Burkholderia spp., constructed with the eBURST algorithm according to allelic profiles obtained from the PubMLST Bcc database. The red circle highlights the clonal complex of B. cepacia CVS136 (node 1986) including isolate PT02 (node 2680). The relative distance between strains indicated above the rib of the graph.

\section{Footnotes}

Author's Contribution: Study concept and design: Irina Zakharova and Dmitry Viktorov; acquisition, analysis, and interpretation of data: Lan Anh Thi Bui, Irina Zakharova, Ivan Shpak, Natalya Teteryatnikova, Dimitriy Ustinov, Yulia Kuzyutina, and Ha Ngoc Nguyen; drafting of the manuscript: Irina Zakharova and Dmitry Viktorov.

Conflict of Interests: Authors declared no conflict of interest.

Funding/Support: This study received no specific financial support.

\section{References}

1. Lipsitz R, Garges S, Aurigemma R, Baccam P, Blaney DD, Cheng AC, et al. Workshop on treatment of and postexposure prophylaxis for Burkholderia pseudomallei and B. mallei Infection, 2010. Emerg InfectDis. 2012;18(12). e2. doi: 10.3201/eid1812.120638. [PubMed: 23171644]. [PubMed Central: PMC3557896].

2. Brent AJ, Matthews PC, Dance DA, Pitt TL, Handy R. Misdiagnosing melioidosis. Emerg Infect Dis. 2007;13(2):349-51. doi: 10.3201/eid1302.061290. [PubMed: 17479916]. [PubMed Central: PMC2725863]

3. Kiratisin P, Santanirand P, Chantratita N, Kaewdaeng S. Accuracy of commercial systems for identification of Burkholderia pseudomallei versus Burkholderia cepacia. Diagn Microbiol Infect Dis. 2007;59(3):277-81. doi: 10.1016/j.diagmicrobio.2007.06.013. [PubMed: 17916419].

4. CLSI. Methods for antimicrobial dilution and disk susceptibility isolated or fastidious bacteria. CLSI guideline M45. 3rd ed. Wayne, PA, USA: Clinical and Laboratory Standards Institute; 2016.
5. Zakharova I, Teteryatnikova N, Toporkov A, Viktorov D. Development of a multiplex PCR assay for the detection and differentiation of Burkholderia pseudomallei, Burkholderia mallei, Burkholderia thailandensis, and Burkholderia cepacia complex. Acta Trop. 2017;174:1-8. doi: 10.1016/j.actatropica.2017.06.016. [PubMed: 28634144].

6. Bankevich A, Nurk S, Antipov D, Gurevich AA, Dvorkin M, Kulikov AS, et al. SPAdes: A new genome assembly algorithm and its applications to single-cell sequencing. J Comput Biol. 2012;19(5):455-77. doi: 10.1089/cmb.2012.0021. [PubMed: 22506599]. [PubMed Central: PMC3342519].

7. Spilker T, Baldwin A, Bumford A, Dowson CG, Mahenthiralingam E, LiPuma JJ. Expanded multilocus sequence typing for Burkholderia species. J Clin Microbiol. 2009;47(8):2607-10. doi: 10.1128/JCM.0077009. [PubMed: 19494070]. [PubMed Central: PMC2725695].

8. Kumar S, Stecher G, Tamura K. MEGA7: Molecular Evolutionary Genetics Analysis Version 7.0 for Bigger Datasets. Mol Biol Evol. 2016;33(7):1870-4. doi: 10.1093/molbev/msw054. [PubMed: 27004904].

9. Deepak RN, Crawley B, Phang E. Burkholderia pseudomallei identification: A comparison between the API 20NE and VITEK2GN systems. Trans R Soc Trop Med Hyg. 2008;102 Suppl 1:S42-4. doi: 10.1016/S00359203(08)70012-9. [PubMed: 19121685].

10. Weissert C, Dollenmaier G, Rafeiner P, Riehm J, Schultze D. Burkholderia pseudomallei misidentified by automated system. Emerg Infect Dis. 2009;15(11):1799-801. doi: 10.3201/eid1511.081719. [PubMed: 19891868]. [PubMed Central: PMC2857215].

11. Podin Y, Kaestli M, McMahon N, Hennessy J, Ngian HU, Wong JS, et al. Reliability of automated biochemical identification of Burkholderia pseudomallei is regionally dependent. J Clin Microbiol. 2013;51(9):3076-8. doi: 10.1128/JCM.01290-13. [PubMed: 23784129]. [PubMed Central: PMC3754659].

12. Zakharova IB, Lopasteyskaya YA, Toporkov AV, Viktorov DV. Influence of biochemical features of Burkholderia pseudomallei strains on identification reliability by vitek 2 system. $J$ Glob Infect Dis. 2018;10(1):7-10. doi: 10.4103/jgid.jgid_39_17. [PubMed: 29563716]. [PubMed Central: PMC5850765]. 
13. Brisse S, Stefani S, Verhoef J, Van Belkum A, Vandamme P, Goessen W. Comparative evaluation of the BD Phoenix and VITEK 2 automated instruments for identification of isolates of the Burkholderia cepacia complex. J Clin Microbiol. 2002;40(5):1743-8. [PubMed: 11980954]. [PubMed Central: PMC130956].

14. Mahenthiralingam E, Bischof J, Byrne SK, Radomski C, Davies JE, Av-Gay Y, et al. DNA-based diagnostic approaches for identification of Burkholderia cepacia complex, Burkholderia vietnamiensis, Burkholderia multivorans, Burkholderia stabilis, and Burkholderia cepacia genomovars I and III. J Clin Microbiol. 2000;38(9):3165-73. [PubMed: 10970351]. [PubMed Central: PMC87345].

15. Jimenez L, Jashari T, Vasquez J, Zapata S, Bochis J, Kulko M, et al. Realtime PCR detection of Burkholderia cepacia in pharmaceutical products contaminated with low levels of bacterial contamination. PDA
J Pharm Sci Technol. 2018;72(1):73-80. doi: 10.5731/pdajpst.2017.007971. [PubMed: 29030528].

16. Suppiah J, Thimma JS, Cheah SH, Vadivelu J. Development and evaluation of polymerase chain reaction assay to detect Burkholderia genus and to differentiate the species in clinical specimens. FEMS Microbiol Lett. 2010;306(1):9-14. doi: 10.1111/j.1574-6968.2010.01923.x. [PubMed: 20345378].

17. Koh SF, Tay ST, Sermswan R, Wongratanacheewin S, Chua KH, Puthucheary SD. Development of a multiplex PCR assay for rapid identification of Burkholderia pseudomallei, Burkholderia thailandensis, Burkholderia mallei and Burkholderia cepacia complex. J Microbiol Methods. 2012;90(3):305-8. doi: 10.1016/j.mimet.2012.06.002. [PubMed: 22705921]. 